\title{
A MATHEMATICAL MODEL OF HIV-1 INFECTION INCLUDING THE SATURATION EFFECT OF HEALTHY CELL PROLIFERATION
}

\author{
MahiÉddine KOUCHE*, BedR'Eddine AINSEBA** \\ * Laboratory of Applied Mathematics \\ Badji-Mokhtar University, BP 12, Annaba 23000, Algeria \\ e-mail: kouchebone@yahoo.fr \\ ** Institute of Mathematics of Bordeaux, UMR CNRS 52 51, Case 36 \\ Université Victor Segalen Bordeaux 2, 3 ter place de la Victoire, F 33076 Bordeaux Cedex, France \\ e-mail: bea@u-bordeaux2.fr
}

\begin{abstract}
In this paper we derive a model describing the dynamics of HIV-1 infection in tissue culture where the infection spreads directly from infected cells to healthy cells trough cell-to-cell contact. We assume that the infection rate between healthy and infected cells is a saturating function of cell concentration. Our analysis shows that if the basic reproduction number does not exceed unity then infected cells are cleared and the disease dies out. Otherwise, the infection is persistent with the existence of an infected equilibrium. Numerical simulations indicate that, depending on the fraction of cells surviving the incubation period, the solutions approach either an infected steady state or a periodic orbit.
\end{abstract}

Keywords: HIV, periodic oscillations, persistence, stability, tissue culture.

\section{Introduction}

Since the early 1980s there has been a tremendous effort made in mathematical modeling of the Human Immunodeficiency Virus type 1 (HIV-1), which causes AIDS (Acquired Immune Deficiency Syndrome). The major target of HIV-1 infection is a class of lymphocytes or white blood cells known as CD4 ${ }^{+}$T-cells, which are the most abundant white blood cells of the immune system. It is thought that HIV-1, although attacking many different cells, wreaks the most havoc on the CD4 ${ }^{+}$T-cells by causing their destruction and decline, and decreasing the body's ability to fight the infection. The process of infection is as follows: First, HIV-1 enters its target T-cell, inside this cell it makes, with the help of the reverse transcriptase enzyme RT, a DNA copy of its viral RNA. The viral DNA is inserted into the DNA of the host cell (Tcell), which will itself produce viral particles that can bud off the cell to infect other healthy cells.

Many mathematical models have been derived in order to describe the dynamics of HIV-1 infection in the bloodstream where cell-free-viral spread is the predominant route of viral spread (De Leenheer and Smith, 2003; Kirschner, 1996; Nowak and Bangham, 1996; Perelson, 1989; Perelson et al., 1993; Perelson and Nelson, 1999;
Nowak and May, 2000). Upon infection with HIV-1, there is a short "intracellular eclipse phase", during which the cell is infected but has not yet begun producing the virus. This intracellular eclipse phase is modeled by introducing an explicit class of latently infected cells. The models are then defined by considering four compartments: cells that are uninfected or healthy cells, cells that are latently infected, cells that are actively infected, and the free virus. The dynamics of these populations are described by a system of four ordinary differential equations. To take into account the "immune responsiveness" of the system, the class of $\mathrm{CD}^{+}{ }^{+} \mathrm{T}$-cells is introduced in the proliferation of CD4 ${ }^{+}$T-cells (Kirschner and Webb, 1996). These cells cannot become infected with the virus, but do destroy infected cells. It was shown that these models can mimic the symptom of AIDS observed clinically such as the long latency period, low levels of the free virus in the body, and the depletion of $\mathrm{CD} 4^{+} \mathrm{T}$-cells.

Herz et al. (1996) proposed to model the latency period by introducing a delay into the class of infected cells by assuming that cells become infected $\tau$ times units after initial infection and obtained a new model of three delay differential equations. Tam (1999) investigated the delay effect in a model which describes the interaction between 
a replicating virus and host cells. Culshaw and Ruan (2000) showed that such an intracellular delay can cause the cell and virus populations to fluctuate in the early stage of infection (called infective oscillations in the literature), and in a long term they will converge to the infected steady state values. Mittler et al. (1998) proposed to model the eclipse phase by a distributed delay assuming that the variation among cells with respect to this intracellular delay can be approximated by a gamma distribution which can mimic a variety of biologically plausible delays. Another type of discrete or distributed delay models has been considered recently in the literature to include antiviral therapy and the effects of drugs (Nelson et al., 2000; Nelson and Perelson, 2002; Wang and Li, 2006).

It was reported that HIV-1 infection is achieved in tissue culture as the lymph nodes and the brain where cellto-cell spread would be a much more important mode of infection than cell-free viral spread (Culshaw et al., 2003; Philips, 1994; Dimitrov et al., 1993). To that end, Spouge et al. (1996) derived a model of HIV-1 cell-to-cell infection kinetics in tissue culture in terms of mathematical models and showed that its asymptotic behavior is similar to that of a model representing cell-free viral spread. The model by Spouge et al. (1996) does not include the latent period after cells have been infected. Culshaw et al. (2003) generalized the model by Spouge et al. (1996) by introducing a distributed delay to model the intracellular eclipse phase. They proved that the intracellular eclipse phase does change the dynamics of the model. More precisely, their results show that, when a fraction of infected cells survives the incubation period and surpasses a critical values, then a Hopf bifurcation occurs inducing periodic fluctuations in cell concentrations.

In the models by Spouge et al. (1996) and Culshaw et al. (2003), it is assumed that HIV-1 infection spreads directly from infected cells to healthy cells by neglecting the free virus and assuming that the infection rate is bilinear, i.e., dependent only on the product of the concentrations of the two cell populations. This last notion, the so-called "mass action principle", is valid when the system is well mixed, i.e., there are no significant spatial concentration heterogeneities, and there are significant quantities of each reactant. However, interactions between two populations are not always so simple, and the form of such an expression can change, for example, to take into account at least three parameters such as the contact rate between infected and healthy cells, the fraction of healthy cells which are activated and hence susceptible to infection, and the fraction of interaction between activated cells and the virus which result in productive infected cells. In a non-wellmixed tissue culture these parameters are not constant and vary, among others, the total size of the populations. It is well known that models for T-cell proliferation are most reasonable when they are based upon a saturation function such that individual T-cells have a well-defined maximal rate of proliferation (De Boer and Perelson, 1995; Callaway and Perelson, 1998).

In this paper we propose a new model of cell-to-cell spread of HIV-1 infection in tissue culture which takes into account the saturation effect of the proliferation of healthy cells. We assume that the infection rate between infected and healthy cells in the model by Culshaw et al. (2003) is a saturating function of cell concentration, i.e.,

$$
\begin{aligned}
& \frac{\mathrm{d} x(t)}{\mathrm{d} t}=r x(t)\left(1-\frac{x(t)+y(t)}{K}\right)-\alpha \frac{x(t) y(t)}{1+q x(t)}, \\
& \frac{\mathrm{d} y(t)}{\mathrm{d} t}=\beta \int_{-\infty}^{t} \frac{f(t-s) x(s) y(s)}{1+q x(s)} \mathrm{d} s-\delta y(t),
\end{aligned}
$$

where $x(t)$, and $y(t)$ denote the concentrations of healthy and infected cells at time $t$, respectively, $r$ is the effective reproductive rate of healthy cells (the term is the total reproductive rate for healthy cells $r_{1}$ minus the death rate for healthy cells $\gamma$ ), $K$ is the effective carrying capacity of the system, $\delta$ is the death rate of infected cells, $\alpha$ is the maximum rate of infection, $\beta$ is such that $\beta / \alpha$ represents the fraction of cells surviving the incubation period, and $1 / q$ denotes the half saturation constant of the proliferation process. The values of these parameters are given in Table 1.

Table 1. Assumed parameter values.

\begin{tabular}{|l|l|l|}
\hline Parameters & Values & Ref. \\
\hline \hline $\begin{array}{l}r \text { healthy cell reproductive } \\
\text { rate }\end{array}$ & $0.68 /$ day & {$[3]$} \\
$K$ carrying capacity of the & $2 \times 10^{6} / \mathrm{mL}$ & {$[3]$} \\
$\begin{array}{l}\delta \text { dem } \\
\text { cells }\end{array}$ & {$[3]$} \\
$\begin{array}{l}k \text { empirical initial growth } \\
\text { of virus } \\
x_{0} \text { initial concentration } \\
\text { of healthy cells } \\
y_{0} \text { initial concentration } \\
\text { of infected cells } \\
\frac{1}{q} \text { half saturation constant } \\
\begin{array}{l}\alpha \text { maximum rate of } \\
\text { infection }\end{array}\end{array}$ & $5 \times 1 /$ day & {$[26]$} \\
\hline
\end{tabular}

The term in the denominator of the infection rate serves to "saturate" the infection rate when $x$ gets large. For example, if $q x \gg 1$, then $\alpha x y /(1+q x) \approx(\alpha / q) y$. In this case the infection rate depends almost exclusively on the number of infected cells and the amount of infected cells present limits the infection rate. A similar saturation term has been used to describe cell growth in models of $\mathrm{T}$ cell proliferation and HIV-1 immune control (Callaway and Perelson, 1998; De Boer and Perelson, 1995; Kirschner and Webb, 1996). We point out that 
as $q \rightarrow 0$, the system (1) is reduced to that described by Culshaw et al. (2003). The model of Culshaw et al. (2003) with the simple mass-action infection term is then an extreme case of the HIV-1 model (1) in this paper. The initial values of the system are

$$
x(s)=\phi(s), \quad y(s)=\psi(s), \quad s \in(-\infty, 0],
$$

with $\phi, \psi \in X$, where $X=C\left((-\infty, 0] ; \mathbb{R}_{+}\right)$is the Banach space of non-negative, continuous and bounded functions on $(-\infty, 0]$. The distributed delay in the second equation of the system (1) models the incubation period by assuming that cells which are productively infectious at time $t$ were infected $\tau$ time units ago, where $\tau$ is the average delay defined by (MacDonald, 1978)

$$
\tau=\int_{0}^{\infty} s f(s) \mathrm{d} s .
$$

In the rest of this paper we take as a delay kernel $f(s)$ the gamma distribution function of order 0 called a weak kernel,

$$
f(s)=\mu e^{-\mu s}, \quad s \geq 0,
$$

for which the average delay is $\tau=1 / \mu$. Such kernels were used in mathematical models of HIV-1 infections by Mittler et al. (1998).

Setting

$$
z(t)=\int_{-\infty}^{t} \mu e^{-\mu(t-s)} \frac{x(s) y(s)}{1+q x(s)} \mathrm{d} s,
$$

we obtain the following system of three ordinary differential equations:

$$
\begin{aligned}
& \frac{\mathrm{d} x(t)}{\mathrm{d} t}=r x(t)\left(1-\frac{x(t)+y(t)}{K}\right)-\alpha \frac{x(t) y(t)}{1+q x(t)}, \\
& \frac{\mathrm{d} y(t)}{\mathrm{d} t}=\mu z^{\prime}(t)-\delta y(t), \\
& \frac{\mathrm{d} z^{\prime}(t)}{\mathrm{d} t}=\beta \frac{x(t) y(t)}{1+q x(t)}-\mu z^{\prime}(t),
\end{aligned}
$$

with the initial values

$$
\begin{aligned}
& x_{0}=\phi(0) \geq 0, \quad y_{0}=\psi(0) \geq 0, \\
& z_{0}=\chi(0)=\int_{-\infty}^{0} \mu e^{\mu s} \frac{\phi(s) \psi(s)}{1+q \phi(s)} \mathrm{d} s \geq 0,
\end{aligned}
$$

where

$$
\chi(s)=\int_{-\infty}^{s} \mu e^{\mu s} \frac{\phi(s) \psi(s)}{1+q \phi(s)} \mathrm{d} s,
$$

$s \leq 0$ and $\phi, \psi \in X$.

To find equilibria of system (2) we set

$$
\begin{aligned}
r x\left(1-\frac{x+y}{K}\right)-\alpha \frac{x y}{1+q x} & =0, \\
\beta z-\delta y & =0, \\
\mu \frac{x y}{1+q x}-\mu z & =0 .
\end{aligned}
$$

It is easy to see that the algebraic system (4) has three equilibrias: the trivial equilibrium $E_{0}=(0,0,0)$, the healthy equilibrium $E_{1}=(K, 0,0)$, and, when

$$
\frac{\beta K}{\delta(1+q K)}>1
$$

there is a third positive equilibrium $E^{*}=\left(x^{*}, y^{*}, z^{*}\right)$, called the infected equilibrium, given by

$$
\begin{aligned}
x^{*} & =\frac{\delta}{\beta-q \delta}, \\
y^{*} & =\frac{r \beta(K \beta-q K \delta-\delta)}{(\beta-q \delta)(\beta r+K \alpha \beta-q K \alpha \delta)}, \\
z^{*} & =\frac{r \delta(K \beta-q K \delta-\delta)}{(\beta-q \delta)(\beta r+K \alpha \beta-q K \alpha \delta)} .
\end{aligned}
$$

In the following section we derive the basic reproduction number $R_{0}$ of the model (2). In Section 3 we prove that, when $R_{0}<1$, the healthy equilibrium $E_{1}$ is locally asymptotically stable, while $E_{0}$ is a saddle point. When $R_{0}>1$, we prove that the infected equilibrium $E^{*}$ exists and is locally asymptotically stable under some hypotheses on the parameters. In Section 4 we are interested in the global dynamics of the model (2). We prove that, when $R_{0} \leq 1$, the healthy equilibrium $E_{1}$ is in fact globally asymptotically stable, and if $R_{0}>1$, we prove that the infection is persistent. By using the average delay as a bifurcation parameter, we prove in this case that the system (2) exhibits two Hopf bifurcations $\mu_{1}$ and $\mu_{2}$, such that the infected equilibrium $E^{*}$ is locally asymptotically stable for $\mu \in\left(0, \mu_{1}\right) \cup\left(\mu_{2}, \infty\right)$, and periodic oscillations occur when $\mu \in\left(\mu_{1}, \mu_{2}\right)$. In Section 5, we report some numerical simulations to illustrate the stability and bifurcation results. As in the work of Culshaw et al. (2003), our model exhibits infective periodic oscillations in realistic parameter regimes, but in our case there are two Hopf bifurcations and the infected steady state is locally asymptotically stable either if the average delay is small enough or sufficiently large.

\section{Basic reproduction number $\boldsymbol{R}_{0}$}

To see whether the virus establishes an infection, a crucial quantity is the basic reproduction number $R_{0}$, which is defined as the number of newly infected cells that arise from any infected cell, over its entire infectious period $1 / \delta$, when most cells are uninfected (Nowak and May, 2000). In epidemiological models, $R_{0}$ is defined as the threshold parameter, such that the disease dies out irrespective of the initial number cases when $R_{0} \leq 1$, and is persistent with the existence of an infected equilibrium when $R_{0}>1$. In this paper, following Diekmann et al. (1990), we derive the basic reproduction number $R_{0}$ for the model (2) by using the method of the next generation matrix, which was modified later by Van den Driessche and Watmough 
(2002), as well as Esteva-Peralta and Velasco-Hernandez (2002).

In order to compute $R_{0}$, we regard (2) as an epidemiological model with three compartments: $x, y$ and $z$. We first write the system (2) the following form:

$$
\begin{aligned}
\frac{\mathrm{d} X}{\mathrm{~d} t} & =F(X)-V(X), \\
V(X) & =V^{-}(X)-V^{+}(X), \\
X & =(x, y, z)^{T},
\end{aligned}
$$

where $F, V^{+}, V^{-}: \mathbb{R}^{3} \rightarrow \mathbb{R}^{3}$ are defined by

$$
\begin{aligned}
F(X) & =\left(0,0, \mu \frac{x y}{1+q x}\right), \\
V^{+}(X) & =\left(r_{1} x\left\{1-\frac{x+y}{K}\right\}, \beta z, 0\right) \\
V^{-}(X) & =\left(\alpha \frac{x y}{1+q x}+\gamma x\left(1-\frac{x+y}{K}\right), \delta y, \mu z\right) .
\end{aligned}
$$

$F(X)$ is the rate of the appearance of new infected cells in each compartment, $V^{+}$is the rate of the transfer of cells into each compartment by all other means, and $V^{-}$is the rate of the transfer of cells out of the compartments. Denote by $\partial F / \partial x, \partial V / \partial x$ the Jacobian matrices of $F$ and $V$ at $E_{1}=(K, 0,0)$, respectively. We have

$$
\begin{aligned}
& \frac{\partial F}{\partial x}=\left(\begin{array}{ccc}
0 & 0 & 0 \\
0 & 0 & 0 \\
0 & \frac{\mu K}{1+q K} & 0
\end{array}\right), \\
& \frac{\partial V}{\partial x}=\left(\begin{array}{ccc}
r_{1}+\gamma & r_{1}+\frac{\alpha K}{1+q K} & 0 \\
0 & \delta & -\beta \\
0 & 0 & \mu
\end{array}\right) .
\end{aligned}
$$

According to (Diekmann et al., 1990) we call $\frac{\partial F}{\partial x}\left(\frac{\partial V}{\partial x}\right)^{-1}$ the next generation matrix of the model (2) and its spectral radius is the corresponding basic reproduction number $R_{0}$. We have

$$
\frac{\partial F}{\partial x}\left(\frac{\partial V}{\partial x}\right)^{-1}=\left(\begin{array}{ccc}
0 & 0 & 0 \\
0 & 0 & 0 \\
0 & \frac{\mu K}{\delta(1+q K)} & \frac{\beta K}{\delta(1+q K)}
\end{array}\right),
$$

and its characteristic polynomial is given by

$$
Q(\lambda)=\lambda^{2}\left(\lambda-\frac{\beta K}{\delta(1+q K)}\right) .
$$

The corresponding eigenvalues are

$$
\lambda_{1,2}=0, \quad \lambda_{3}=\frac{\beta K}{\delta(1+q K)} .
$$

The spectral radius of the next generation matrix $\frac{\partial F}{\partial x}\left(\frac{\partial V}{\partial x}\right)^{-1}$ is then $\beta K / \delta(1+q K)$. According to Diekmann et al. (1990), we define the basic reproduction number $R_{0}$ for model (2) by

$$
R_{0}=\frac{1}{\delta}\left[\frac{\beta K}{1+q K}\right] .
$$

The term in brackets describes the newly infected cells that arise from any infected cell when almost all cells are uninfected. We will prove in Section 4 that the dynamics of the system (2) are largely determined by the basic reproduction number $R_{0}$.

\section{Local stability of equilibria}

In this section we analyze the local stability of the equilibria of the system (2). Denote by $J(E)$ the Jacobian matrix of the system (2) at $E=(x, y, z)$. We have

$$
J(E)=\left(\begin{array}{ccc}
r-2 r \frac{x}{K}-r \frac{y}{K}-\frac{\alpha y}{(1+q x)^{2}} & \\
0 & & \\
\frac{\mu y}{(1+q x)^{2}} & \\
-r \frac{x}{K}-\alpha \frac{x}{1+q x} & 0 \\
-\delta & \beta \\
\mu \frac{x}{1+q x} & -\mu
\end{array}\right) .
$$

Theorem 1. $E_{0}$ is a saddle point and $E_{1}$ is locally asymptotically stable provided that $R_{0}<1$.

Proof. Denote by $P(\lambda)$ the characteristic polynomial of the system (2) at $(x, y, z)$. We have, after some calculations, that

$$
\begin{aligned}
P(\lambda)= & \lambda^{3}+(\delta+\mu-A) \lambda^{2}+(\delta \mu-A \delta-A \mu \\
& \left.-\beta \frac{\mu x}{1+q x}\right) \lambda+\frac{A \beta \mu x}{1+q x} \\
& +B \frac{\beta \mu y}{(1+q x)^{2}}-A \mu \delta,
\end{aligned}
$$

where

$$
\begin{aligned}
& A=r-\frac{r}{K} y-2 \frac{r}{K} x-\frac{\alpha y}{(1+q x)^{2}}, \\
& B=\frac{r}{K} x+\frac{\alpha x}{1+q x} .
\end{aligned}
$$

Case 1. Trivial equilibrium $E_{0}=(0,0,0)$ :

Set $x=y=z=0$. The characteristic polynomial (5) takes the form

$$
\begin{aligned}
P(\lambda)= & \lambda^{3}+(\delta+\mu-r) \lambda^{2}+(\delta \mu-r \delta-r \mu) \lambda \\
& -r \mu \delta \\
= & (r-\lambda)(\lambda+\mu)(\lambda+\delta),
\end{aligned}
$$

and therefore $P$ has two negative real roots $\lambda_{1}=-\delta$, $\lambda_{2}=-\mu$ and one positive real root $\lambda_{3}=r>0$. The trivial equilibrium is a saddle point.

Case 2. Healthy equilibrium $E_{1}=(K, 0,0)$ : 
In this case the characteristic polynomial has the form

$$
\begin{aligned}
P(\lambda)= & \lambda^{3}+(r+\mu+\delta) \lambda^{2}+(r \delta+r \mu \\
& \left.+\mu\left(\delta-\frac{\beta K}{1+q K}\right)\right) \lambda \\
& +r \mu\left(\delta-\frac{\beta K}{1+q K}\right) \\
= & \lambda^{3}+(r+\mu+\delta) \lambda^{2} \\
& +\left(r \delta+r \mu+\frac{\mu}{\delta}\left(1-R_{0}\right)\right) \lambda \\
& +r \frac{\mu}{\delta}\left(1-R_{0}\right) .
\end{aligned}
$$

Since $R_{0}<1$, all the coefficients of $P(\lambda)$ are positive, and the inequality

$$
(r+\mu+\delta)\left[r \delta+r \mu+\frac{\mu}{\delta}\left(1-R_{0}\right)\right]>r \frac{\mu}{\delta}\left(1-R_{0}\right)
$$

holds. So by the Routh-Hurwitz theorem all the roots of $P(\lambda)$ have negative real parts. The healthy equilibrium $E_{1}$ is locally asymptotically stable.

We now turn to proving the local stability of the infected equilibrium $E^{*}$. We showed in Section 2 that, if $R_{0}>1$, then the infected equilibrium exists. In the following theorem we give some hypotheses on the parameters under which $E^{*}$ is locally asymptotically stable.

The Jacobian matrix $J\left(E^{*}\right)$ of (2) at point $E^{*}=$ $\left(x^{*}, y^{*}, z^{*}\right)$ is

$$
J\left(E^{*}\right)=\left(\begin{array}{ccc}
-a & -b & 0 \\
0 & -\delta & \beta \\
\frac{\mu y^{*}}{\left(1+q x^{*}\right)^{2}} & \mu \frac{\delta}{\beta} & -\mu
\end{array}\right),
$$

where

$$
\begin{aligned}
& a=-r+y^{*} \frac{r}{K}+2 \frac{r}{K} x^{*}+\frac{\alpha y^{*}}{\left(1+q x^{*}\right)^{2}}, \\
& b=r \frac{x^{*}}{K}+\alpha \frac{\delta}{\beta} .
\end{aligned}
$$

To proceed further, we make the following assumption on the parameters of the system (2):

$$
r-2 r \frac{x^{*}}{K}-r \frac{y^{*}}{K}-\frac{\alpha y^{*}}{\left(1+q x^{*}\right)^{2}}<0 .
$$

Write

$$
\begin{aligned}
& \Delta=\left[a-\frac{b}{a} \frac{\beta y^{*}}{\left(1+q x^{*}\right)^{2}}\right]^{2}-4 \delta \frac{b}{a} \frac{\beta y^{*}}{\left(1+q x^{*}\right)^{2}}, \\
& D=2 \delta+a-\frac{b}{a} \frac{\beta y^{*}}{\left(1+q x^{*}\right)^{2}} .
\end{aligned}
$$

Theorem 2. Assume that $R_{0}>1$, and (6) holds. (i) If either $\Delta<0$ or $\Delta=0$ and $D>0$, then $E^{*}$ is locally asymptotically stable for all $\mu>0$.

(ii) If $\Delta=0$ and $D<0$, there is a $\mu_{0}>0$, such that $E^{*}$ is locally asymptotically stable for $\mu>0$ and $\mu \neq \mu_{0}$ and unstable for $\mu=\mu_{0}$.

(iii) If $\Delta>0$, we have the following two cases:

1. $D>0$, then $E^{*}$ is locally asymptotically stable for all $\mu>0$.

2. $D<0$, then there are $0<\mu_{1}<\mu_{2}$, such that $E^{*}$ is locally asymptotically stable for $\mu<\mu_{1}$ or $\mu>\mu_{2}$, and is unstable for $\mu \in\left(\mu_{1}, \mu_{2}\right)$. Further, the system (2) exhibits Hopf bifurcations at $\mu=\mu_{1}$ and $\mu=\mu_{2}$.

Proof. Let $P(\lambda)$ be the characteristic polynomial of the Jacobian matrix $J\left(E^{*}\right)$. We have

$$
\begin{aligned}
P(\lambda)= & \lambda^{3}+(a+\mu+\delta) \lambda^{2}+(a \mu+a \delta) \lambda \\
& +b \frac{\mu \beta y^{*}}{\left(1+q x^{*}\right)^{2}} .
\end{aligned}
$$

From(6) it follows that $a>0$ and all the coefficients of $P(\lambda)$ are positive. Applying the Routh-Hurwitz theorem to (7), we find that $E^{*}$ is locally asymptotically stable if and only if

$$
\begin{gathered}
A(\mu)=(a+\delta+\mu)(a \delta+a \mu) \\
-b \frac{\mu \beta y^{*}}{\left(1+q x^{*}\right)^{2}}>0 .
\end{gathered}
$$

Taking $\mu>0$ as a bifurcation parameter, we rewrite (8) into the following form:

$$
\begin{aligned}
A(\mu) & =\mu^{2}+\left[2 \delta+a-\frac{b}{a} \frac{\beta y^{*}}{\left(1+q x^{*}\right)^{2}}\right] \mu+\delta^{2}+a \delta \\
& =\mu^{2}+D \mu+\delta^{2}+a \delta
\end{aligned}
$$

for which the discriminant is $\Delta$. We then have the following cases:

- If $\Delta<0$, or $\Delta=0$ and $D>0$, then $A(\mu)$ has no real roots or one negative real root, and in this case $A(\mu)>0$ while $E^{*}$ is locally asymptotically stable for all $\mu>0$.

- If $\Delta=0$ and $D<0, A(\mu)$ has one positive real root $\mu_{0}$ and $A(\mu)>0$ for all $\mu>0$ and $\mu \neq \mu_{0}$. $E^{*}$ is then locally asymptotically stable for all $\mu>0$, and $\mu \neq \mu_{0}$ and unstable for $\mu=\mu_{0}$.

- Assume now that $\Delta>0$. Then, if $D>0, A(\mu)$ has two negative real roots, so $A(\mu)>0$. In this case the infected equilibrium $E^{*}$ is locally asymptotically stable for all $\mu>0$.

- When $D<0, A(\mu)$ has two positive real roots $0<$ $\mu_{1}<\mu_{2}$ such that $A(\mu)>0$, for $\mu \in\left(0, \mu_{1}\right) \cup$ 
$\left(\mu_{2},+\infty\right)$ and $A(\mu)<0$, for $\mu \in\left(\mu_{1}, \mu_{2}\right) . E^{*}$ is then locally asymptotically stable for $\mu \in\left(0, \mu_{1}\right) \cup$ $\left(\mu_{2},+\infty\right)$ and unstable for $\mu \in\left(\mu_{1}, \mu_{2}\right)$. Further,

$$
\begin{aligned}
& \frac{\mathrm{d} A\left(\mu_{1}\right)}{\mathrm{d} \mu}=2 \mu_{1}+D=\sqrt{\Delta}>0, \\
& \frac{\mathrm{d} A\left(\mu_{2}\right)}{\mathrm{d} \mu}=2 \mu_{2}+D=-\sqrt{\Delta}<0 .
\end{aligned}
$$

Let $\lambda(\mu)=\eta(\mu)+i \omega(\mu)$ be the eigenvalue of $P(\lambda)$, where $\eta(\mu)$ and $\omega(\mu)$ depend on $\mu$. We first show that for $\mu=\mu_{1,2}, P(\lambda)$ has one negative real root and two conjugate purely imaginary roots. Since

$$
\left(a+\delta+\mu_{1,2}\right)\left(a \delta+a \mu_{1,2}\right)=b \frac{\mu_{1,2} \beta y^{*}}{\left(1+q x^{*}\right)^{2}},
$$

we have

$$
P(\lambda)=\left(\lambda+a+\delta+\mu_{1,2}\right)\left(\lambda^{2}+a \delta+a \mu_{1,2}\right) .
$$

Hence $P(\lambda)$ has one negative real root $\lambda_{1}=-(a+$ $\left.\delta+\mu_{1,2}\right)<0$ and two conjugate purely imaginary roots $\lambda_{2,3}= \pm i \sqrt{a \delta+a \mu_{1,2}}$. Substituting $\lambda(\mu)=\eta(\mu)+$ $i \omega(\mu)$ into (7), we find that

$$
\begin{array}{r}
\eta^{3}-3 \eta \omega^{2}+3 i \eta^{2} \omega-i \omega^{3}+(a+\delta+\mu)\left(\eta^{2}-\omega^{2}\right) \\
+2 i(a+\delta+\mu) \eta \omega+\eta(a \delta+a \mu)+i \omega(a \delta+a \mu) \\
+b \frac{\mu \beta y^{*}}{\left(1+q x^{*}\right)^{2}}=0 .
\end{array}
$$

Separating the real and imaginary parts, we obtain

$$
\begin{gathered}
\eta^{3}-3 \eta \omega^{2}+(a+\delta+\mu)\left(\eta^{2}-\omega^{2}\right)+\eta(a \delta+a \mu) \\
+b \frac{\mu \beta y^{*}}{\left(1+q x^{*}\right)^{2}}=0 .
\end{gathered}
$$

Differentiating the expression in (10) with respect to $\mu$ and using the fact that $\eta\left(\mu_{1,2}\right)=0$ and $\omega^{2}\left(\mu_{1,2}\right)=$ $a \delta+a \mu_{1,2}$, we get

$$
\begin{array}{r}
-\left.3\left(a \delta+a \mu_{1,2}\right) \frac{\mathrm{d} \eta}{\mathrm{d} \mu}\right|_{\mu=\mu_{1,2}} \\
-\left.\frac{\mathrm{d}}{\mathrm{d} \mu}(a \delta+a \mu)(a+\delta+\mu)\right|_{\mu=\mu_{1,2}} \\
+\left.\frac{\mathrm{d}}{\mathrm{d} \mu}\left(b \frac{\mu \beta y^{*}}{\left(1+q x^{*}\right)^{2}}\right)\right|_{\mu=\mu_{1,2}}=0 .
\end{array}
$$

Hence we obtain, with the help of (8) and (9),

$$
\begin{aligned}
-\left.3\left(a \delta+a \mu_{1,2}\right) \frac{\mathrm{d} \eta}{\mathrm{d} \mu}\right|_{\mu=\mu_{1,2}} & =\left.\frac{\mathrm{d} A(\mu)}{\mathrm{d} \mu}\right|_{\mu=\mu_{1,2}} \\
& = \pm \sqrt{\Delta} \neq 0,
\end{aligned}
$$

thus a family of periodic solutions bifurcates from $E^{*}$ when $\mu$ passes trough one of the critical values $\mu_{1}$ or $\mu_{2}$ (Hassard et al., 1981).

\section{Global dynamics of the model}

In this section we analyze the global asymptotic behavior of the system (2). We begin with the following lemma on the dissipativity of (2).

Lemma 1. The positive orthant is positively invariant by the system (2) and there exists a constant $M>0$ independent of the solutions such that

$$
\begin{aligned}
& \limsup _{t \rightarrow+\infty} x(t) \leq K, \quad \limsup _{t \rightarrow+\infty} y(t) \leq M, \\
& \limsup _{t \rightarrow+\infty} z(t) \leq M .
\end{aligned}
$$

Proof. The invariance of the positive orthant is trivial. We have to prove the ultimate boundedness of the solutions. We have, from the first equation of the system (2),

$$
\frac{\mathrm{d} x(t)}{\mathrm{d} t} \leq r x(t)\left(1-\frac{x(t)}{K}\right),
$$

which gives, with the help of the comparison principle,

$$
\limsup _{t \rightarrow+\infty} x(t) \leq K .
$$

Define the function

$$
W(t)=x(t)+\frac{\alpha}{\mu} z(t) .
$$

We have

$$
\begin{aligned}
\frac{\mathrm{d} W}{\mathrm{~d} t} & \leq r x-\alpha z \\
& \leq(r+\mu) x-\mu x-\alpha z \\
& \leq(r+\mu) x-\mu W(t) .
\end{aligned}
$$

Let $\epsilon>0$ be fixed. By (11), there is $T^{\epsilon}>0$ such that $x(t) \leq K+\epsilon$ for $t>T^{\epsilon}$. Combining this last relation with (12), for $t>T^{\epsilon}$ we have

$$
\frac{\mathrm{d} W}{\mathrm{~d} t}(t) \leq(r+\mu)(K+\epsilon)-\mu W(t),
$$

which leads to

$$
\limsup _{t \rightarrow+\infty} W(t) \leq \frac{K(r+\mu)}{\mu} .
$$

Finally, from the second equation in (2) and the fact that $z$ is uniformly bounded it follows that there is an

$$
M>\frac{K(r+\mu)}{\mu}
$$

such that

$$
\limsup _{t \rightarrow+\infty} y(t) \leq M .
$$

This completes the proof of the lemma. 
In Section 3 we proved that, if $R_{0}<1$, the healthy equilibrium $E_{1}$ is locally asymptotically stable. In the following theorem we prove that $E_{1}$ is globally asymptotically stable when $R_{0} \leq 1$.

Theorem 3. Assume that $R_{0} \leq 1$. Then the healthy equilibrium $E_{1}$ is globally stable.

Proof. We analyze separately two cases: $R_{0}<1$ and $R_{0}=1$.

Case 1. $R_{0}<1$

Define the set $G=\{(\phi, \psi, \chi) \in X: 0 \leq \phi \leq K, \psi, \chi \geq$ $0\}$. By Lemma 1, $G$ attracts all solutions of the system (2). Let $(\phi, \psi, \chi) \in G$, and denote by $(x(t), y(t), z(t))$ the corresponding solution of (2). We claim that $x(t) \leq K$ for all $t>0$. Otherwise, there is $t_{1}>0$ such that $x\left(t_{1}\right)=K$ and $\mathrm{d} x\left(t_{1}\right) / \mathrm{d} t>0$. We have

$$
\begin{aligned}
\frac{\mathrm{d} x}{\mathrm{~d} t}\left(t_{1}\right) & \leq r x\left(t_{1}\right)\left(1-\frac{x\left(t_{1}\right)+y\left(t_{1}\right)}{K}\right) \\
& \leq-r y\left(t_{1}\right) \\
& \leq 0,
\end{aligned}
$$

which contradicts the fact that $\mathrm{d} x\left(t_{1}\right) / \mathrm{d} t>0$. Thus $G$ is positively invariant with respect to (2). For any $(\phi, \psi, \chi) \in G$, we define the functional $V(\phi, \psi, \chi)=$ $\mu y+\beta z$, where $(x(t), y(t), z(t))$ is the corresponding solution to (2). From the system (2) we have that

$$
\frac{\mathrm{d} V}{\mathrm{~d} t}=\left(\beta \frac{\mu x}{1+q x}-\delta \mu\right) y .
$$

Since $G$ is positively invariant and $R_{0}<1$, we have $x(t) \leq K$ for all $t>0$. Hence (13) leads to

$$
\begin{aligned}
\frac{\mathrm{d} V}{\mathrm{~d} t} \leq \mu\left\{\frac{\beta K}{1+q K}\right. & -\delta\} y \\
& =\frac{\mu}{\delta}\left(R_{0}-1\right) y<0, \quad t>0 .
\end{aligned}
$$

$V$ is then a Lyapunov functional on $G$. Define now the set

$$
E=\left\{(\phi, \psi, \chi) \in G: \frac{\mathrm{d} V}{\mathrm{~d} t}(\phi, \psi, \chi)=0\right\},
$$

and let $M$ be the largest set in $E$ which is invariant with respect to (2). Clearly, $M$ is not empty since $E_{1}=$ $(K, 0,0) \in M$. Let $(\phi, \psi, \chi) \in M$ and $(x(t), y(t), z(t))$ be the solution of (2) with the initial data $(\phi, \psi, \chi)$. From the invariance of $M$, we have $\left(x_{t}, y_{t}, z_{t}\right) \in M$ for all $t \in R$. Thus $\mathrm{d} V / \mathrm{d} t=0$, and, from (13), $y(t)=0$ for all $t \in R$. We obtain from the first and the third equations in (2) that $x(t) \rightarrow K$ and $z(t) \rightarrow 0$ as $t \rightarrow+\infty$. The invariance of $M$ implies that $x(t)=K$ and $z(t)=0$ for all $t \in R$. Therefore, $M=\{(K, 0,0)\}=E_{1}$. Since it was shown in Theorem 1 that $E_{1}$ is locally asymptotically stable when $R_{0}<1$, from the LaSalle invariance principle it follows that $E_{1}$ is globally asymptotically stable.
Case 2. $R_{0}=1$

By the first equation of (2), $x(t)$ is always decreasing when above $K$. We can prove that if there exists some $t_{0}>0$ such that $x\left(t_{0}\right)<K$, then $x(t)<K$ for all $t>t_{0}$. Otherwise, there must exist some $t_{1}>t_{0}$ such that $x\left(t_{1}\right)=K$ and $\mathrm{d} x\left(t_{1}\right) / \mathrm{d} t \geq 0$. This is impossible. Hence, there are two possible cases:

(i) $x(t)>K$, for all $t>0$ and $x(t) \rightarrow K$ as $t \rightarrow+\infty$.

(ii) There exists $t_{0}>0$ such that $x\left(t_{0}\right)<K$.

In Case (ii) we have that $x(t)<K$ for all $t>t_{0}$. The global stability of $E_{1}$ can proved by using the same Lyapunov functional $V$ as in the first part of the proof.

In Case (i) we rewrite the first equation in (2) as follows:

$$
r \frac{x y}{K} \leq r x\left(1-\frac{x}{K}\right)-\frac{\mathrm{d} x}{\mathrm{~d} t} .
$$

Now, since $x(t)>K$, from the preceding equation we obtain

$$
r y(t) \leq-\frac{\mathrm{d} x}{\mathrm{~d} t}, \quad t>0 .
$$

Integrating the last relation over $(0, t)$, we find that

$$
r \int_{0}^{t} y(s) \mathrm{d} s \leq x(0)-x(t) \leq x(0), \quad t>0
$$

which implies, with the boundedness of $y(t)$, that $y(t) \rightarrow$ 0 , and thus $z(t) \rightarrow 0$ as $t \rightarrow+\infty$. $E_{1}$ is then globally stable.

Theorem 3 completely determines the global dynamics of the system when $R_{0} \leq 1$ and can be interpreted as follows: If $R_{0} \leq 1$, then the infection will not spread since every infected cell will on the average produce less than one new infected cell. The chain reaction is sub-critical. In the following theorem, we will prove that, if $R_{0}>1$, then a chain reaction takes place and leads to a chronic infection.

Before giving the second main result of this section, we recall some results about the theory of infinite dimensional systems (Thieme, 1993). Let $X$ be a metric space with metric $d$. Assume that $X$ is the union of two disjoint subsets $X_{1}$ and $X_{2}$. A continuous semiflow on $X_{1}$ is a continuous mapping $\Phi:\left[0, \infty\left[\times X_{1} \rightarrow X_{1}\right.\right.$ such that

$$
\Phi_{t} \circ \Phi_{s}=\Phi_{t+s}, \quad t, s \geq 0, \quad \Phi_{0}(x)=x, \quad \forall x \in X_{1},
$$

where $\Phi_{t}$ denotes the mapping from $X_{1}$ to $X_{1}$ given by

$$
\Phi_{t}(x)=\Phi(t, x) .
$$

We say that a subset $K$ of $X$ is a weak repeller of $X_{1}$ if there an is $\epsilon>0$ such that

$$
\limsup _{t \rightarrow \infty} d\left(\Phi_{t}(x), K\right)>\epsilon, \quad \forall x \in X_{1} .
$$


$K$ is called a uniform strong repeller for $X_{1}$ if there is an $\epsilon>0$ such that

$$
\liminf _{t \rightarrow \infty} d\left(\Phi_{t}(x), K\right)>\epsilon, \quad \forall x \in X_{1} .
$$

Lemma 2. (Thieme, 1993) Let $X_{1}$ be open in $X$ and the semiflow $\Phi$ be dissipative, i.e, there is a bounded set $B \subset X$ such that $d\left(\Phi_{t}(x), B\right) \longrightarrow 0$ for all $x \in X_{1}$. Further, assume that $A_{\partial}$,

$$
\begin{aligned}
A_{\partial} & =\bigcup_{y \in Y} \omega(y), \\
Y & =\left\{x \in X_{2}: \Phi_{t}(x) \in X_{2}, \forall t>0\right\},
\end{aligned}
$$

has an acyclic isolated covering $M=\bigcup_{k=1}^{m} M_{k}$ such that each $M_{k}$ is a weak repeller for $X_{1}$. Then $X_{2}$ is a uniform strong repeller for $X_{1}$.

Theorem 4. Assume that $R_{0}>1$. Then the infection is persistent.

Proof. Recall that solutions of the system (2) are said to be persistent (or uniformly persistent) if there is an $\epsilon>$ 0 such that, for all solutions $(x(t), y(t), z(t))$ of (2) for which $x(0), y(0), z(0)>0$, we have

$$
\liminf _{t \rightarrow+\infty} x(t) \geq \epsilon, \quad \liminf _{t \rightarrow+\infty} y(t) \geq \epsilon, \quad \liminf _{t \rightarrow+\infty} z(t) \geq \epsilon .
$$

To this end we use the persistence theory for infinite dimensional systems (Thieme, 1993). In order to apply Theorem 4 of (Thieme, 1993), we define the sets

$$
\begin{aligned}
X & =\left\{(x, y, z) \in \mathbb{R}^{3}: x, y, z \geq 0\right\}, \quad X_{1}=\operatorname{int}(X), \\
X_{2} & =\left\{(x, y, z) \in \mathbb{R}^{3}: x=0 \text { or } z=0\right\} .
\end{aligned}
$$

We have $X=X_{1} \cup X_{2}$ and $X_{1} \cap X_{2}=\emptyset$. Let $U=\{(x, y, z) \in X: x \leq K, y \leq M, z \leq M\}$. From Lemma 1 , it is easy to see that $U$ is a compact absorbing set. The semiflow associated with the system (2) is then dissipative. Denote by $\omega\left(x_{0}, y_{0}, z_{0}\right)$ the omega limit set of the solution to the system (2) starting at $\left(x_{0}, y_{0}, z_{0}\right) \in X$ (which exists by Lemma 1). We have to determine the following set:

$$
A_{\partial}=\bigcup_{\left(x_{0}, y_{0}, z_{0}\right) \in Y} \omega\left(x_{0}, y_{0}, z_{0}\right),
$$

where

$$
Y=\left\{\left(x_{0}, y_{0}, z_{0}\right) \in X_{2}: \Phi_{t}\left(x_{0}, y_{0}, z_{0}\right) \in X_{2}, \forall t>0\right\},
$$

in which $\Phi_{t}\left(x_{0}, y_{0}, z_{0}\right)$ denotes the solution semiflow of the system (2) starting at $\left(x_{0}, y_{0}, z_{0}\right)$. It follows from Lemma 1 that $X_{1}$ is an invariant set with respect to the semiflow of the system (2).

Let $\left(x_{0}, y_{0}, z_{0}\right) \in \partial X_{2}$.There are two distinct cases: 1. $x_{0}=0$. In this case we have, from the first equation of the system (2),

$$
\frac{\mathrm{d} x}{\mathrm{~d} t} \leq r x\left(1-\frac{x}{K}\right), \quad x(0)=0,
$$

which implies that $x(t)=0$, for all $t>0$. The third equation of the system (2) gives $z(t) \rightarrow 0$ as $t \rightarrow+\infty$, and hence $y(t) \rightarrow 0$ as $t \rightarrow+\infty$. So the omega limit set becomes $\omega\left(x_{0}, y_{0}, z_{0}\right)=\left\{E_{0}\right\}$.

2. $z_{0}=0$. Then, by (3), either $x(0)=0$ or $y(0)=0$. In the first case, we have $x_{0}=0$, which implies, according to the first part of the proof, that $\omega\left(x_{0}, y_{0}, z_{0}\right)=\left\{E_{0}\right\}$. If $y(0)=0$, then by the second and third equations of the system (2) we obtain $y(t), z(t) \rightarrow 0$ as $t \rightarrow+\infty$, and by the first one $x(t) \rightarrow K$ as $t \rightarrow+\infty$. Thus $\omega\left(x_{0}, y_{0}, z_{0}\right)=$ $\left\{E_{1}\right\}$. Hence $A_{\partial}=\left\{E_{0}\right\} \cup\left\{E_{1}\right\}$ and $\left\{E_{0}\right\},\left\{E_{1}\right\}$ are an acyclic isolated covering for $A_{\partial}$.

It remains to prove that $\left\{E_{0}\right\},\left\{E_{1}\right\}$ are weak repellers for $X_{1}$, i.e., $W^{s}\left(E_{i}\right) \cap X_{1}=\emptyset$ for $i=0,1$, where

$$
W^{s}\left(E_{i}\right)=\left\{\left(x_{0}, y_{0}, z_{0}\right) \in X: \omega\left(x_{0}, y_{0}, z_{0}\right)=E_{i}\right\}
$$

is the stable manifold of $E_{i}$. Assume that $W^{s}\left(E_{0}\right) \cap X_{1} \neq$ $\emptyset$. Then there is a solution $(x(t), y(t), z(t))$ to the system (2) such that $(x(t), y(t), z(t)) \rightarrow(0,0,0)$ as $t \rightarrow+\infty$. Hence from the first equation of the system (2), there is a $T_{0}>0$ such that

$$
\frac{\mathrm{d}(\ln x)}{\mathrm{d} t} \geq r\left\{1-\frac{x}{K}\right\}-\alpha \frac{y}{1+q x} \geq \frac{r}{2}, \quad t>T_{0},
$$

which implies that $x(t) \rightarrow+\infty$ as $t \rightarrow+\infty$, which is a contradiction.

In the second case assume, on the contrary, that there holds $W^{s}\left(E_{1}\right) \cap X_{1} \neq \emptyset$. There is then a solution $(x(t), y(t), z(t))$ of the system (2) such that $(x(t), y(t), z(t)) \rightarrow(K, 0,0)$ as $t \rightarrow+\infty$. Since $R_{0}>1$, there are $\epsilon>0$ and $T_{1}>0$ such that

$$
\begin{aligned}
& x(t)>K-\epsilon, \quad t>T_{1}, \\
& \frac{\beta(K-\epsilon)}{1+q(K-\epsilon)}>\delta .
\end{aligned}
$$

From the system (2) and the first equation in (15), we have

$$
\begin{gathered}
\frac{\mathrm{d} y}{\mathrm{~d} t}=\beta z-\delta y, \\
\frac{\mathrm{d} z}{\mathrm{~d} t} \geq \mu \frac{(K-\epsilon)}{1+q(K-\epsilon)} y-\mu z .
\end{gathered}
$$

The second inequality in (15) implies

$$
\begin{aligned}
& \frac{\mathrm{d}}{\mathrm{d} t}(\mu y(t)+\beta z(t)) \\
& \quad=\mu y(t)\left(\frac{\beta(K-\epsilon)}{1+q(K-\epsilon)}-\delta\right)>0
\end{aligned}
$$

for all $t>T_{1}$. We deduce that $\mu y(t)+\beta z(t)>\mu y\left(T_{1}\right)+$ $\beta z\left(T_{1}\right)>0$ for all $t>T_{1}$, which contradicts the fact that $y(t), z(t) \rightarrow 0$ as $t \rightarrow+\infty$. By Lemma $2, X_{2}$ is a uniform strong repeller for $X_{1}$. This means that solutions of system (2) are uniformly persistent. 
In the rest of this section we investigate the dynamics of the model (2) when $E^{*}$ is unstable. In the following Theorem, we use the generalized Poincaré-Bendixon theorem to prove that, if the infected equilibrium is unstable, then periodic oscillations of cell concentrations may occur.

Let $x \mapsto f(x) \in \mathbb{R}^{n}$ be a smooth vector field for $x$ in an open subset $\Omega \subset \mathbb{R}^{n}$. The differential equation

$$
x^{\prime}=f(x), \quad x \in \Omega,
$$

is said to be competitive in $\Omega$ if, for some diagonal matrix $H=\operatorname{diag}\left(\epsilon_{1}, \ldots, \epsilon_{n}\right)$, where each $\epsilon_{i}$ is either 1 or -1 , the matrix $H\left(\frac{\partial f}{\partial x}\right) H$ has non-positive off-diagonal elements for all $x \in \Omega$, where $\frac{\partial f}{\partial x}$ denotes the Jacobian matrix of $f$. The following result is a consequence of the PoincaréBendixon theorem.

Theorem 5. Assume that $R_{0}>1$ and (6) holds. Then any non-empty omega limit set $L$ of the system (2) either converges to $E^{*}$ or is a non-trivial periodic orbit. Further, if $E^{*}$ is unstable, then every solution except those with initial data on the stable manifold of $E^{*}$ or on $\partial X^{0}$ approaches a non-trivial periodic orbit.

Proof. Define

$$
\Omega=\left\{(x, y, z) \in \mathbb{R}^{3}: x>0, y>0, z>0\right\} .
$$

Clearly, $\Omega$ is an open convex set, and by Lemma 1 , the system (2) is dissipative. Since $R_{0}>1$, by Theorem 5 each orbit $(x(t), y(t), z(t))$ starting in $\Omega$ enters the compact set

$$
\{(x, y, z) \in \Omega: \epsilon \leq x \leq K, \epsilon \leq y, z \leq M\}
$$

for $t$ sufficiently large, and the only equilibrium point in $\Omega$ is the infected equilibrium $E^{*}$.

Define the matrix $H$ as follows:

$$
H=\left(\begin{array}{ccc}
1 & 0 & 0 \\
0 & 1 & 0 \\
0 & 0 & -1
\end{array}\right)
$$

Then

$$
\begin{aligned}
& H J(E) H=\left(\begin{array}{c}
r-2 r \frac{x}{K}-r \frac{y}{K}-\frac{\alpha y}{(1+q x)^{2}} \\
0 \\
-\frac{\mu y}{(1+q x)^{2}}
\end{array}\right. \\
& \left.\begin{array}{ccc}
-r \frac{x}{K}-\alpha \frac{x}{1+q x} & 0 & \\
-\delta & -\beta & \\
& -\mu \frac{x}{1+q x} & -\mu
\end{array}\right),
\end{aligned}
$$

where $J(E)$ is the Jacobian matrix of the system (2) at $E=(x, y, z)$. Thus, by (16), the matrix $H J(E) H$ has all its off-diagonal elements non-positive for all $E \in \Omega$. The system (2) is then competitive in $\Omega$. Further, we have, at the infected equilibrium $E^{*}$,

$$
\begin{aligned}
\operatorname{det} & J\left(E^{*}\right) \\
& =\operatorname{det}\left(\begin{array}{ccc}
-a & -b & 0 \\
0 & -\delta & \beta \\
\frac{\mu y^{*}}{\left(1+q x^{*}\right)^{2}} & \mu \frac{\delta}{\beta} & -\mu
\end{array}\right) \\
& =-b \beta \frac{\mu y^{*}}{\left(1+q x^{*}\right)^{2}}<0 .
\end{aligned}
$$

By the generalized Poincaré-Bendixon theorem (Zhu and Smith, 1994), we conclude that each orbit starting in $\Omega$ converges to the infected equilibrium $E^{*}$ or is a non-trivial periodic orbit.

To prove the last part of the theorem, it suffices to prove that the Jacobian matrix $J\left(E^{*}\right)$ has two eigenvalues with positive real parts and one negative eigenvalue (Smith, 1995). Assume that $E^{*}$ is unstable. We first prove that $J\left(E^{*}\right)$ has one negative eigenvalue $\lambda_{1}$. By (16), the matrix $-H J\left(E^{*}\right) H$ has all its elements non-negative. By the Perron-Frobenius theorem $-H J\left(E^{*}\right) H$ has one real positive eigenvalue and thus the matrix $H J\left(E^{*}\right) H$ has one negative eigenvalue. Since $H J\left(E^{*}\right) H$ and $J\left(E^{*}\right)$ have the same eigenvalues, we conclude that $J\left(E^{*}\right)$ has one negative eigenvalue, which we denote by $\lambda_{1}$. Denote by $\lambda_{2}=u_{2}+i v_{2}$ and $\lambda_{3}=u_{3}+i v_{3}$ the other two eigenvalues. The characteristic polynomial $P(\lambda)$ of $J\left(E^{*}\right)$ can be written in the following form:

$$
P(\lambda)=\left(\lambda-\lambda_{1}\right)\left(\lambda-\lambda_{2}\right)\left(\lambda-\lambda_{3}\right) .
$$

Now, since the coefficients of zero and second powers of $\lambda$ in $P(\lambda)$ are positive (see (7)), it follows that the term $u_{2} u_{3}+v_{2}^{2}+i\left(u_{2}-u_{3}\right) v_{2}$ is real and positive. Thus, $u_{2}=$ $u_{3}$, which implies with the fact that $E^{*}$ is unstable, that $u_{2}>0$ and $u_{3}>0$. This completes the proof.

The following corollary is a direct consequence of Theorem 5 .

Corollary 1. Under the second hypothesis of Part (iii) in Theorem 2 and for $\mu \in\left(\mu_{1}, \mu_{2}\right)$, each orbit of the system (2) except those with initial data on the stable manifold of $E^{*}$ or on $X_{2}$ approaches a non-trivial periodic orbit.

\section{Numerical simulations}

In this section we report some numerical simulations of the model (1) to illustrate our results on stability and bifurcations. To carry out the numerical simulations, we have to determine the model parameters. Since the parameters which do not depend on the infection process such as $r, K, \delta$ are known and taken from the literature (see Table 1), it remains to determine the unknown parameters, 
which are the maximal infected rate $\alpha$ and the half saturation constant of the proliferation process $1 / q$. There are no empirical values of these parameters in the literature. However, we propose their estimates. Firstly, we use the fact that the basic reproduction number $R_{0}$, which represents the number of newly infected cells produced by one infected cell at the beginning of the infection, can be computed by using the parameter values given by Culshaw et al. (2003). If we assume that all cells survive the incubation period, we find that $R_{0}$ is around 13 . We assume that this number is approximately the same as in our model. Further, by Section 2 and the assumption that all cells survive the incubation period (i.e., $\alpha=\beta$ ), we know that $R_{0}=\frac{\alpha K}{\delta(1+q K)}$, and then

$$
\frac{\alpha K}{\delta(1+q K)} \simeq 13
$$

Secondly, it is well known that, for HIV-1 cell-to-cell spread, the initial exponential growth of infected cells $k$ is a linear function of the initial concentration of infected cells (Spouge et al., 1996; Dimitrov et al., 1993) and is around 1 per day. Since the exponential growth of infected cells at time $t=0$ is given by $y_{0}^{-1} \mathrm{~d} y(0) / \mathrm{d} t$, we obtain from the second equation in (2) that $k \simeq \alpha z_{0} / y_{0}-\delta$. If we assume now that the initial concentrations of healthy and infected cells are constants around $x_{0}=5 \times 10^{5}$ cells $/ \mathrm{mL}$ and $y_{0}=5 \times 10^{2}$ cells $/ \mathrm{mL}$, respectively, we obtain that

$$
k \simeq \frac{\alpha x_{0}}{1+q x_{0}}-\delta .
$$

Knowing $K, x_{0}, \delta$ and $k$ (see Table 1 ) we can compute $q$ and $\alpha$ from Eqns. (17) and (18), and we find that $q=$ $2.2 \times 10^{-7} / \mathrm{mL}$ and $\alpha=2.84 \times 10^{-6} /$ day.

Suppose that $50 \%$ of infected cells survive incubation, which corresponds to $\beta=1.42 \times 10^{-6}$. Then $R_{0}=6.25, a=0.05, \Delta=7.33, D=-2.78$. The second condition of Part (iii) in Theorem 2 is then satisfied. The critical parameters are $\mu_{1}=0.038, \mu_{2}=2.74$. If we take as the average delay $\mu=1$, which is a realistic value since the incubation period is around 1 day (Spouge et al., 1996), then Corollary 1 predicts that the solution to the system (1) approaches a periodic solution. Figure 1 shows that in this case periodic oscillations of cell concentrations occur with a period around 25 days.

Assume now that $32 \%$ of infected cells survive incubation, which corresponds to $\beta=0.9 \times 10^{-6}$. We obtain the following values: $R_{0}=4.16, a=0.086$, $\Delta=0.77$ and $D=-1.11$. The corresponding values are $\mu_{1}=0.11, \mu_{2}=0.99$, which is biologically realistic for $\mu_{2}$. If we take $\mu=0.9$, we obtain Fig. 2, which shows periodic oscillations in cell concentrations. If we increase $\mu_{2}$ to the value of 1.1 , numerical simulations give the plots in Fig. 3, which shows damped oscillations of cell concentrations followed by convergence to the infected steady state.
Finally, assume that $25 \%$ of infected cells survive incubation, corresponding to $\beta=0.71 \times 10^{-6}$. We have in this case $R_{0}=3.25, a=0.11>0, \Delta=-0.23<0$. Condition (i) in Theorem 2 is satisfied and the theorem predicts that the infected equilibrium $E^{*}$ is locally asymptotically stable. Numerical simulations (see Fig. 4) show that in this case the concentrations of healthy and infected cells converge to the infected equilibrium $E^{*}$.
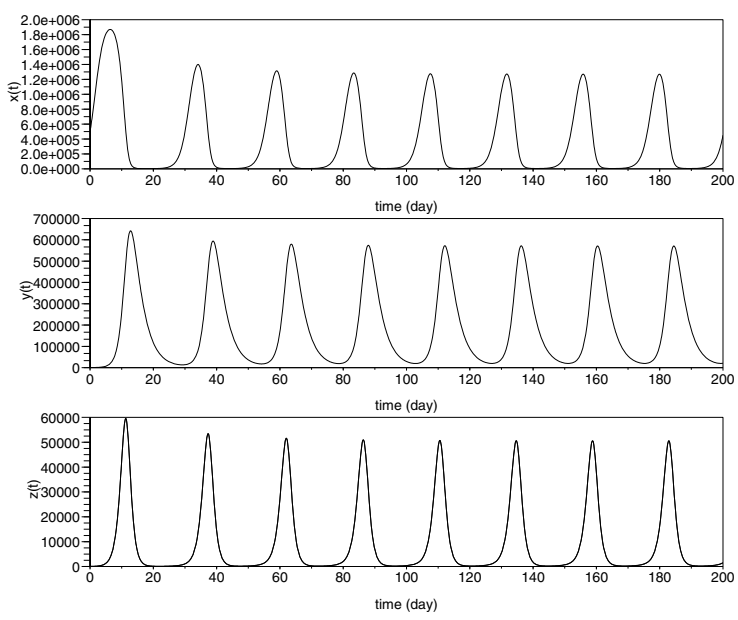

Fig. 1. Half infected cells survive incubation: $\mu=1$.
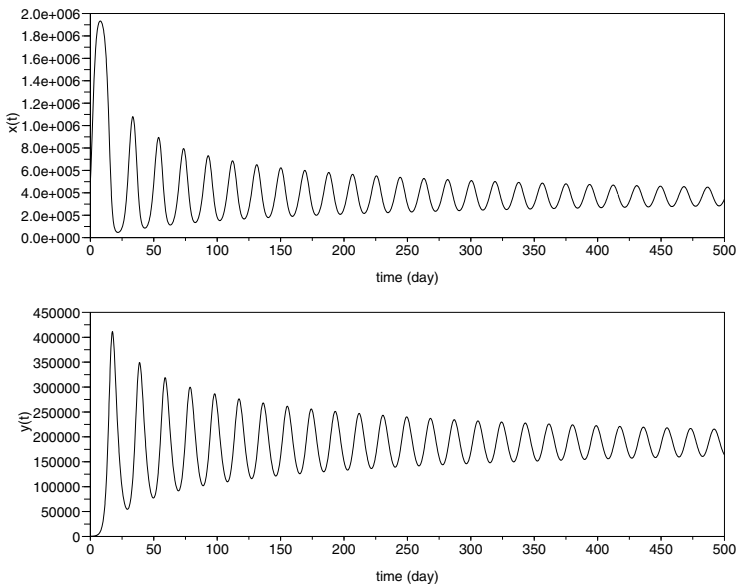

Fig. 2. $32 \%$ of infected cells survive incubation: $\mu=0.9$.

\section{Discussion}

It is well known that HIV-1 is thought to be active in areas such as the brain and the lymph nodes, where $98 \%$ of lymphocytes reside and in which cell-to-cell spread would be a much more important mode of infection than cell-free viral spread since viral replication in a system with rapid cell turnover kinetics depends on cell-to-cell transfer of virus (Culshaw et al., 2003; Dimitrov et al., 1993; Spouge et al., 1996).

In this paper we modified the infection rate between infected and healthy cells in the model proposed by $\mathrm{Cul}-$ 

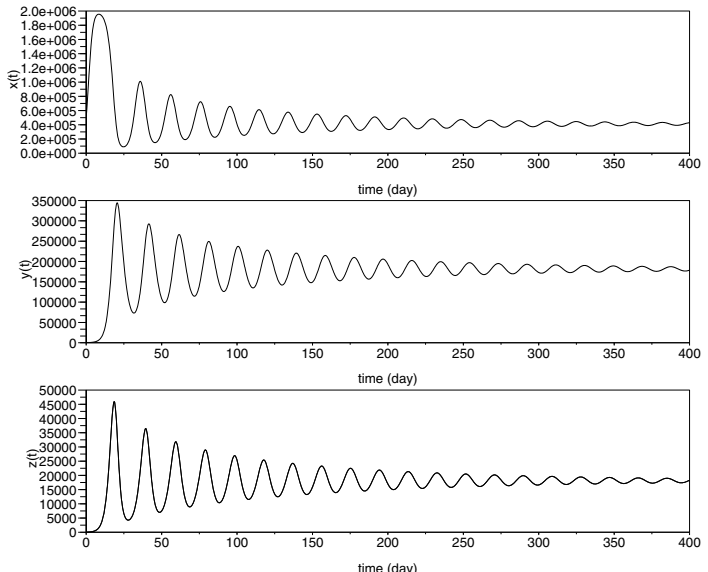

Fig. 3. $32 \%$ of infected cells survive incubation: $\mu=1.1$.
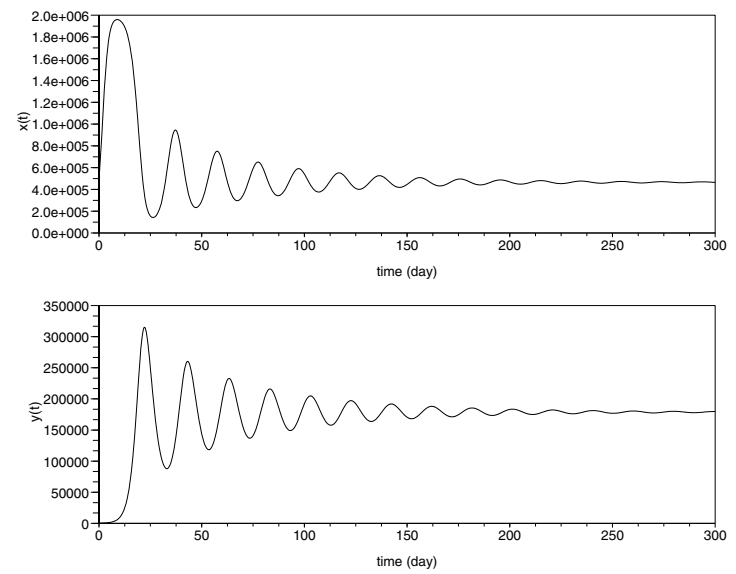

Fig. 4. $25 \%$ of infected cells survive incubation.

shaw et al. (2003) by assuming that the infection rate term is a saturating function of cell concentration. By using the method of the next generation matrix, the basic reproduction number $R_{0}$ of the model is derived. We also proved that the model has at most three equilibria: trivial, healthy and, in case $R_{0}>1$, there is a third equilibrium called the infected equilibrium. Stability analysis shows that, when $R_{0} \leq 1$, infected cells dies out and the healthy equilibrium is globally stable. When $R_{0}>1$, we proved that the infection is persistent. When the infected equilibrium is unstable, infective oscillations may occur but, unlike the model of Culshaw et al. (2003), the system exhibits two Hopf bifurcations $\mu_{1}$ and $\mu_{2}$ such that the infected equilibrium is locally asymptotically stable either when the average delay is sufficiently large $\left(\mu>\mu_{2}\right)$ or small $\left(\mu<\mu_{1}\right)$.

Numerical simulations show that, if no more than $7.7 \%$ of infected cells survive the incubation period, infected cells die out and the system converges to its healthy equilibrium. If between $7.7 \%$ and $30 \%$ of infected cells survive the incubation, then the system stabilizes at its in- fected equilibrium, and when more than $30 \%$ of infected cells survive the incubation, then we observe periodic oscillations in cell concentrations. Further, an increase in the fraction of cells surviving the incubation $\beta / \alpha$, would increase the periods and amplitudes of cell concentrations, and a reduction in such a fraction leads to damped oscillations and eventually steady states.

Qualitatively, our model differs from that of Culshaw et al. (2003) since under realistic parameter regimes it exhibits two Hopf bifurcations and the infected steady state is locally asymptotically stable either when the average delay is sufficiently large or small. For the other values of the delay, the model exhibits stable periodic solutions. The existence of stable periodic solutions is due to the third compartment, which is the compartment of latently infected cells, since the system with only two compartments (healthy and infected cells) doest not generate a periodic solution. This result confirm the experimental work of Grossman et al. (1998), which showed that infected and latently infected cells may not contribute significantly to the viral load but may be instrumental in sustaining the infection (Spouge et al., 1996).

Quantitatively, we observe in our model some overestimates in the periods of cell concentrations and the critical bifurcation value of cells surviving the incubation period. If $75 \%$ of infected cells survive the incubation, our model indicates a period of around 30 days of cell concentrations and a critical bifurcation value around 30 , for equivalent values of around 25 days and $22.5 \%$, respectively, in the model by Culshaw et al. (2003).

The model proposed in this paper is a first step in studying cell-to-cell HIV-1 spread in non-well-mixed tissue culture. Another compartmental models can be considered to take into account more significantly spatial heterogeneities in tissue culture. We leave this for a future work.

\section{Acknowledgment}

The authors would like to thank Prof. F.A. Milner of Purdue University (USA) for helpful discussions and comments. The first author also wishes to thank the Laboratory of Applied Mathematics of Bordeaux (MAB) for its financial support.

\section{References}

Callaway, D.S. and Perelson, A.S. (1998). HIV-1 infection and low steady state viral loads, Bulletin of Mathematical Biology 64(5): 29-64.

Culshaw, R. and Ruan, S. (2000). A delay-differential equation model of hiv infection of $\mathrm{CD} 4^{+}$T-cells, Mathematical Biosciences 165(1): 27-39.

Culshaw, R., Ruan, S. and Webb, G. (2003). A mathematical model of cell-to-cell spread of HIV-1 that includes a time delay, Journal of Mathematical Biology 46(5): 425-444. 
De Boer, R.J. and Perelson, A.S. (1995). Towards a general function describing T-cell proliferation, Journal of Theoretical Biology 175(4): 567-576.

De Leenheer, P. and Smith, H.L. (2003). Virus dynamics: A global analysis, SIAM Journal of Applied Mathematics 63(4): 1313-1327.

Diekmann, O., Heesterbeek, J.A.P. and Metz, J. (1990). On the definition and the computation of the basic reproduction ratio $R_{0}$ in models for infectious diseases in heterogeneous populations, Journal of Mathematical Biology 29(4): 365382.

Dimitrov, D.S., Willey, R.L., Sato, H., Chang, L.J., Blumenthal, R. and Martin, M.A. (1993). Quantitation of human immunodeficiency virus type 1 infection kinetics, Journal of Virology 67(4): 2182-2190.

Esteva-Peralta, L. and Velasco-Hernandez, J.X. (2002). M-matrices and local stability in epidemic models, Mathematical and Computer Modelling 36(4-5): 491-501.

Grossman, Z., Feinberg, M.B. and Paul, W. (1998). Multiple modes of cellular activation and virus transmission in HIV infection: A role for chronically and latently infected cells in sustaining viral replication, Proceedings of the $\mathrm{Na}$ tional Academy of Sciences of the United States of America 95(11): 6314-6319.

Hassard, B.D., Kazarinoff, N.D. and Wan, Y.H. (1981). Theory and Applications of Hopf Bifurcation, Cambridge University Press, Cambridge.

Herz, A. V.M., Bonhoeffer, S., Anderson, R.M., May, R.M. and Nowak, M. A. (1996). Viral dynamics in vivo: Limitations on estimates of intracellular delay and virus decay, Proceedings of the National Academy of Sciences of the United States of America 93(14): 7247-7251.

Kirschner, D. E. (1996). Using mathematics to understand HIV immune system, Notices of the American Mathematical Society 43(2): 191.

Kirschner, D.E. and Webb, G. (1996). A model for treatment strategy in the chemotherapy of AIDS, Bulletin of Mathematical Biology 58(2): 167-190.

MacDonald, N. (1978). Time Delays in Biological Models, Springer-Verlag, New York, NY.

Mittler, J.E., Sulzer, B., Neumann, A.U. and Perelson, A.S. (1998). Influence of delayed viral production on viral dynamics in HIV-1 infected patients, Mathematical Biosciences 152(2): 143-163.

Nelson, P.W., Murray, J.D. and Perelson, A.S. (2000). A model of HIV-1 pathogenesis that includes an intracellular delay, Mathematical Biosciences 163(2): 201-215.

Nelson, P.W. and Perelson, A.S. (2002). Mathematical analysis of delay differential equation models of HIV-1 infection, Mathematical Biosciences 179(1): 73-94.

Nowak, M.A. and Bangham, R.M. (1996). Population dynamics of immune responses to persistent viruses, Science 272(5258): 74-79.
Nowak, M.A. and May, R.M. (2000). Virus Dynamics, Oxford University Press, New York, NY.

Perelson, A.S. (1989). Modelling the interaction of the immune system with HIV, in C. Castillo-Chavez (Ed.), Mathematical and Statistical Approaches to AIDS Epidemiology, Springer-Verlag, New York, NY, pp. 350-370.

Perelson, A.S., Kirschner, D.E. and Boer, R.D. (1993). Dynamics of HIV infection of $\mathrm{CD}^{+}{ }^{+}$T-cells, Mathematical Biosciences 114(1): 81-125.

Perelson, A.S. and Nelson, P.W. (1999). Mathematical analysis of HIV-1 dynamics in vivo, SIAM Review 41(1): 3-44.

Philips, D. M. (1994). The role of cell-to-cell transmission in HIV infection, AIDS 8(6): 719-731.

Smith, H.L. (1995). Monotone Dynamical Systems, American Mathematical Society, Providence, RI.

Spouge, J.L., Shrager, R.I. and Dimitrov, D.S. (1996). HIV-1 infection kinetics in tissue cultures, Mathematical Biosciences 138(1): 1-22.

Tam, J. (1999). Delay effect in a model for virus replication, IMA Journal of Mathematics Applied to Medicine and Biology 16(1): 29-37.

Thieme, H.R. (1993). Persistence under relaxed pointdissipativity (with application to an endemic model), SIAM Journal on Mathematical Analysis 24(2): 407.

van den Driessche, P. and Watmough, J. (2002). Reproduction numbers and sub-threshold endemic equilibria for compartmental models of disease transmission, Mathematical Biosciences 180(1-2): 29-48.

Wang, L. and Li, M.Y. (2006). Mathematical analysis of the global dynamics of a model for HIV infection of $\mathrm{CD}^{+}$ T-cells, Mathematical Biosciences 200(1): 4-57.

Zhu, H. and Smith, H.L. (1994). Stable periodic orbits for a class of three dimensional competitive systems, Journal of Differential Equations 110(1): 143-156.

Mahiéddine Kouche was born in Annaba, Algeria, in 1970. He obtained his doctorate in applied mathematics from Badji-Mokhtar University in 2007. His research interests include delay differential equations, dynamical systems, and mathematical biology. Currently he is an assistant professor at the Department of Mathematics of Badji-Mokhtar University.

Bedr'eddine Ainseba was born in Tlemcen, Algeria, in 1968. He received his Ph.D. from Compiègne University in 1992. Presently he is professor at the University of Bordeaux. His current interests involve control theory, age structured systems, dynamical systems, and mathematical biology.

Received: 31 May 2009

Revised: 28 March 2010 